\title{
EDEMA CEREBRAL CRÔNICO NA NEUROCISTICERCOSE
}

\author{
SVETLANA AGAPEJEV*, DANIELA ANGERAME YELA**, ANDRÉ EDUARDO GOMES **
}

\begin{abstract}
RESUMO - Neste estudo retrospectivo, relatam-se as características clínicas do edema cerebral crônico (ECCr) em 34 pacientes com neurocisticercose $(\mathrm{NCC})$, que apresentavam edema cerebral difuso, à tomografia computadorizada (TC), como característica comum. Todos foram tratados com dextroclorofeniramina e, 32 deles, com albendazol. O ECCr predominou no sexo feminino $(73,5 \%)$ na faixa etária dos 11 - 40 anos $(92,3 \%)$. A cefaléia ocorreu em $94,1 \%$ dos pacientes, náuseas/vômitos em $47,1 \%$, crises epilépticas em $41,1 \%$ e distúrbios psíquicos em $38,2 \%$. A hiperreflexia ocorreu em $82,3 \%$ e o papiledema em $58,8 \%$ e o exame neurológico normal em 11,8\%. Na TC, o edema esteve associado a calcificações em 61,8\% dos casos. As pressões liquóricas foram mais elevadas $(\mathrm{p}<0,05)$ antes do tratamento. Atualmente, estão assintomáticos, ou com melhora clínica, 79,4\% dos pacientes ( $57,1 \%$ deles sem medicação). Discute-se a possibilidade do ECCr, na NCC, ser uma manifestação antigênica, sem a presença concomitante de cistos parasitários, e poder representar mais uma condição clínica associada à hipertensão intracraniana benigna.
\end{abstract}

PALAVRAS-CHAVE: neurocisticercose, edema cerebral crônico, hipertensão intracraniana benigna, tratamento.

\section{Chronic brain oedema in neurocysticercosis}

ABSTRACT - In a retrospective study, we report the clinical characteristics of chronic brain oedema (CBO) in 34 patients with neurocysticercosis (NCC) who presented diffuse brain oedema upon computed tomography (CT) as a common point. All patients received dextrochlorpheniramine, and, $94.1 \%$ of them also albendazole. A predominance of females $(73.5 \%)$ aged $11-40$ years $(92.3 \%)$ was seen. Headache occurred in $94.1 \%$ of patients, nausea/vomiting in $47.1 \%$, epileptic manifestations in $41.1 \%$ and psychiatric disorders in $38.2 \%$. Hyperreflexia was obtained in $82.3 \%$ of patients, papilloedema in $58.8 \%$ and normal neurological examination in $11.8 \%$. The CT scan of the brain showed an association of oedema with calcifications in $61.8 \%$ of cases. The cerebrospinal fluid (CSF) pressure was higher before than after treatment $(\mathrm{p}<0.05)$. At the last visit, $79.4 \%$ of patients were currently asymptomatic (57.1\% of them without medication). It is pointed out that $\mathrm{CBO}$ may be an antigenic manifestation in NCC without the concomitant presence of parasitic cysts and may represent an additional clinical condition associated with benign intracranial hypertension.

KEY WORDS: neurocysticercosis, brain oedema, benign intracranial hypertension, treatment.

A neurocisticercose (NCC) é a doença parasitária mais comum do sistema nervoso central (SNC), causada pela forma larvária da Taenia solium. Os sinais e sintomas decorrentes dessa infecção dependem, fundamentalmente, da resposta imunológica do hospedeiro ${ }^{1-3} \mathrm{e}$, como resultado, mostram variabilidade em suas exteriorizações clínicas.

As manifestações desta neuroparasitose ocorrem através de múltiplas facetas, variando desde simples enxaqueca ${ }^{4}$ aos mais graves sinais e sintomas de hipertensão intracraniana ${ }^{5}$, passando por

*Professora Assistente Doutora, docente do Departamento de Neurologia e Psiquiatria da Faculdade de Medicina da Universidade Estadual Paulista (UNESP), Botucatu (SP); ** $6^{\circ}$ anistas de Medicina da UNESP. Aceite: 3-julho-1998.

Dra. Svetlana Agapejev - Departamento de Neurologia e Psiquiatria, Faculdade de Medicina, UNESP - Caixa Postal 540 - 18603-970 Botucatu SP - Brasil. Email: agapejev@ brain.fmb.unesp.br 
crises epilépticas apenas, ou caracterizando quadros clínicos complexos. A NCC pode ser assintomática, mesmo na forma racemosa e/ou de localização intraventricular ${ }^{2}$, ou ser fatal, como nos doentes com hipertensão intracraniana (HIC) e que apresentam a clássica síndrome do líquido cefalorraqueano (LCR) na $\mathrm{NCC}^{6}$ ou, até mesmo, nas suas exteriorizações mais benignas como as calcificações em doentes epilépticos mas que desenvolvem o estado de mal epiléptico. Tudo depende de uma imprevisível interação: a resposta do hospedeiro ao parasita e a resposta do parasita às reações do hospedeiro.

Este estudo tem como objetivo apresentar as características de mais uma das formas de manifestação da NCC, o edema cerebral crônico (ECCr).

\section{PACIENTES E MÉTODOS}

Foram selecionados 34 pacientes seguidos no Serviço de Neurologia do Hospital das Clínicas da Faculdade de Medicina de Botucatu (HCFMB), que apresentavam queixas crônicas, sinais de edema cerebral difuso (Tabela 1, item A) nos exames de TC de crânio e preenchessem 3, ou mais, critérios de inclusão para o diagnóstico de NCC (Tabela 1, item B). Estes pacientes foram estudados, retrospectivamente, através da análise de seus prontuários, quanto às queixas apresentadas desde a $1^{\underline{a}}$ consulta, evolução clínica e alterações em exames complementares: LCR, tomografia computadorizada (TC), hemograma, glicemia, $\mathrm{Ca}^{++}, \mathrm{Mg}^{++}, \mathrm{Na}^{+}, \mathrm{K}^{+}$, e sorologia para lues e toxoplasmose.

Todos os pacientes foram tratados com dextroclorofeniramina (DCF) de liberação lenta (12-18 mg/dia, em 2 - 3 tomadas, via oral), desde antes da administração do albendazol (ABZ) (15 - $30 \mathrm{mg} / \mathrm{kg} / \mathrm{dia}$, em 3 tomadas, via oral, por 21 - 30 dias). Apenas 2 pacientes não receberam ABZ. Esse esquema terapêutico, utilizado no Serviço de Neurologia do HCFMB desde 1984, foi associado a acetazolamida (250-500 mg/dia, em 2 tomadas, via oral) e ao cetoprofeno (150-300 mg/dia, em 1 a 3 tomadas, via oral). Pacientes com síndrome epiléptica receberam anticonvulsivantes, nas dosagens habituais, desde a primeira consulta.

Não foi viável a realização de ressonância magnética nuclear, em qualquer dos pacientes, antes do esquema terapêutico proposto.

Para a comparação das variáveis do LCR (glicose, proteínas, celularidade global e específica, pressão inicial e final) e do sangue (hemograma, glicemia, $\mathrm{Ca}^{++}, \mathrm{Mg}^{++}, \mathrm{Na}^{+}, \mathrm{K}^{+}$), nas situações antes e depois do tratamento instituído, utilizou-se o teste de Wilcoxon.

\section{RESULTADOS}

Os critérios de inclusão (Tabela 1) foram aplicáveis em 9 homens e 25 mulheres. No sexo masculino, a idade variou de 16 a 38 anos (mediana $=22$ anos, $\bar{x} \pm s d=23,7 \pm 7,1$ anos) e predomínio na faixa etária dos 11-20 anos (55,5\%). Nas mulheres, a idade variou de 14 a 53 anos (mediana $=27$ anos, moda $=18$ anos, $\overline{\mathrm{x}} \pm \mathrm{sd}=28,8 \pm 10,1$ anos), e predomínio de $11-40$ anos $(92,3 \%)$, principalmente,

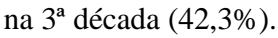

\section{Tabela 1. Critérios de inclusão.}

A. Sinais tomográficos de edema cerebral difuso:

1. Apagamento dos sulcos cerebrais;

2. Diminuição no diâmetro ventricular, principalmente dos laterais e III ${ }^{\circ}$;

3. Precária diferenciação entre substância cerebral branca e cinzenta.

B. Critério para diagnóstico de neurocisticercose:

1. Procedência de área reconhecidamente endêmica ${ }^{6}$;

2. Síndrome liquórica clássica ${ }^{7}$ ou positividade isolada das reações imunológicas para cisticercose no LCR, indiferentemente se antes, durante ou após tratamento com albendazol;

3. TC de crânio com lesões sugestivas de cisticercos em qualquer etapa de seu desenvolvimento;

4. Resposta terapêutica satisfatória após o uso do albendazol, do ponto de vista de exames complementares (TC, LCR) e dos sinais e sintomas. 
Tabela 2. Sinais e sintomas apresentados pelos 34 pacientes com edema cerebral crônico pela neurocisticercose durante a primeira consulta.

\begin{tabular}{lcc}
\hline Sinais e sintomas & Número de pacientes & $\%$ \\
\hline Cefaléia & 32 & 94,1 \\
Hiperreflexia* & 28 & 82,3 \\
Papiledema & 20 & 58,8 \\
Náuseas / vômitos & 16 & 47,1 \\
Crises epilépticas & 14 & 41,1 \\
Distúrbios psíquicos & 13 & 38,2 \\
Tontura & 6 & 17,6 \\
Distúrbios endócrinos & 5 & 14,7 \\
Sinais vestibulares & 5 & 14,7 \\
Embaçamento visual & 4 & 11,8 \\
Exame neurológico normal & 4 & 11,8 \\
Parestesias & 3 & 8,8 \\
Rigidez de nuca & 3 & 8,8 \\
Síndrome do cordão posterior & 1 & 2,9 \\
\hline
\end{tabular}

*A hiperreflexia foi universal em $16(57,1 \%)$ pacientes, apenas aquiliana em $6(21,4 \%)$, apenas nos membros inferiores em $5(17,9 \%)$ e apenas nos membros superiores em um $(3,6 \%)$.

Na Tabela 2 estão expressas as queixas e os sinais neurológicos apresentados pelos 34 pacientes. Essas queixas estiveram associadas entre si, em 32 deles $(94,1 \%)$. Apenas 2 pacientes tiveram a cefaléia como única manifestação. Das 6 mulheres que fizeram uso de anticoncepcionais, a cefaléia iniciou-se durante a vigência deles em $5(83,3 \%)$ e não diminuiu com sua suspensão. As características da cefaléia não foram adequadamente definidas por 40,6\%. Nos demais, foi latejante em $11(34,4 \%)$, em pontadas em $4(12,5 \%)$ ou em peso em $4(12,5 \%)$, de localização holocraniana em $13(40,6 \%)$, regional em $14(43,7 \%)$ ou errante em $5(15,6 \%)$, de aparecimento matinal em todos e, nas mulheres, com piora no período pré-menstrual. As manifestações epilépticas, relatadas em 14 pacientes, clinicamente, foram parciais em $5(35,7 \%)$, parciais com generalização secundária em $8(57,1 \%)$ e generalizadas em apenas um $(7,1 \%)$. Os sintomas psíquicos, excluindo aqueles inerentes ao período pós-crise epiléptica, estiveram sempre associados a outros sintomas em todos os 13 pacientes e entraram no contexto das queixas principais, traduzindo-se por irritabilidade em $6(46,1 \%)$, nervosismo em $5(38,5 \%)$, depressão em $3(23,1 \%)$ e ansiedade em $2(15,4 \%)$. Das 5 pacientes com queixas endócrinas, 2 eram portadoras de ovário policístico, outras 2 referiam secreção leitosa nas mamas e uma apresentava manifestações sugestivas de tireoidopatia.

A fundoscopia óptica foi normal em 8 pacientes que, também, apresentavam hiperreflexia universal. A intensidade do papiledema foi variável. A rigidez de nuca foi detectada em 2 pacientes com pleocitose no LCR e em um outro com LCR mostrando apenas hiperproteinorraquia. Nenhum paciente mostrou presença de sinais neurológicos localizatórios.

A TC de crânio caracterizou-se por edema cerebral difuso de discreto a intenso, em todos os pacientes, associando-se a outras lesões em 87,9\% deles (Tabela 3). O aspecto arredondado do IVo ventrículo esteve presente em 27,3\% e, em 33,3\%, acompanhado de calcificações (Fig 1). Nas últimas TCs desses pacientes, o edema cerebral havia regredido, parcial ou totalmente, dependendo do tempo de evolução ser inferior ou superior a 4-5 anos. O mesmo foi observado em relação ao IVo ventrículo arredondado. 
Tabela 3. Alterações tomográficas apresentadas no primeiro exame dos 34 pacientes com edema cerebral crônico pela neurocisticercose.

\begin{tabular}{lcc}
\hline Alterações tomográficas & Número de pacientes & $\%$ \\
\hline Edema cerebral* $^{*}$ & 34 & 100,0 \\
Múltiplas calcificações & 13 & 38,2 \\
IVo ventrículo arredondado & 9 & 26,5 \\
Calcificação única & 8 & 23,5 \\
Possíveis vesículas & 6 & 17,6 \\
\hline
\end{tabular}

*O edema cerebral, como única alteração, ocorreu em 4 (11,8\%) pacientes.

O exame do primeiro LCR obtido, em todos, através de punção suboccipital cisternal, mostrou celularidade normal em 79,4\% dos pacientes e aumento em 20,6\%, às custas de pleocitose do tipo linfomonocitária, com 1 a $6 \%$ de eosinófilos. Os valores da proteinorraquia, da glicorraquia e da celularidade, excluindo os $20,6 \%$ dos pacientes com pleocitose, não apresentaram diferença estatisticamente significante $(\mathrm{p}>0,05)$ entre antes e depois do tratamento cisticida. As reações imunológicas para cisticercose (fixação de complemento e/ou imunofluorescência) foram positivas em $11,8 \%$ dos 34 pacientes (em um paciente com pleocitose e em 3 com LCR normal). As pressões liquóricas iniciais $\left(118 \pm 85 \mathrm{mmH}_{2} \mathrm{O}\right)$ e finais $\left(63 \pm 22 \mathrm{mmH}_{2} \mathrm{O}\right)$ foram maiores $(\mathrm{p}<0,05)$ antes do tratamento.

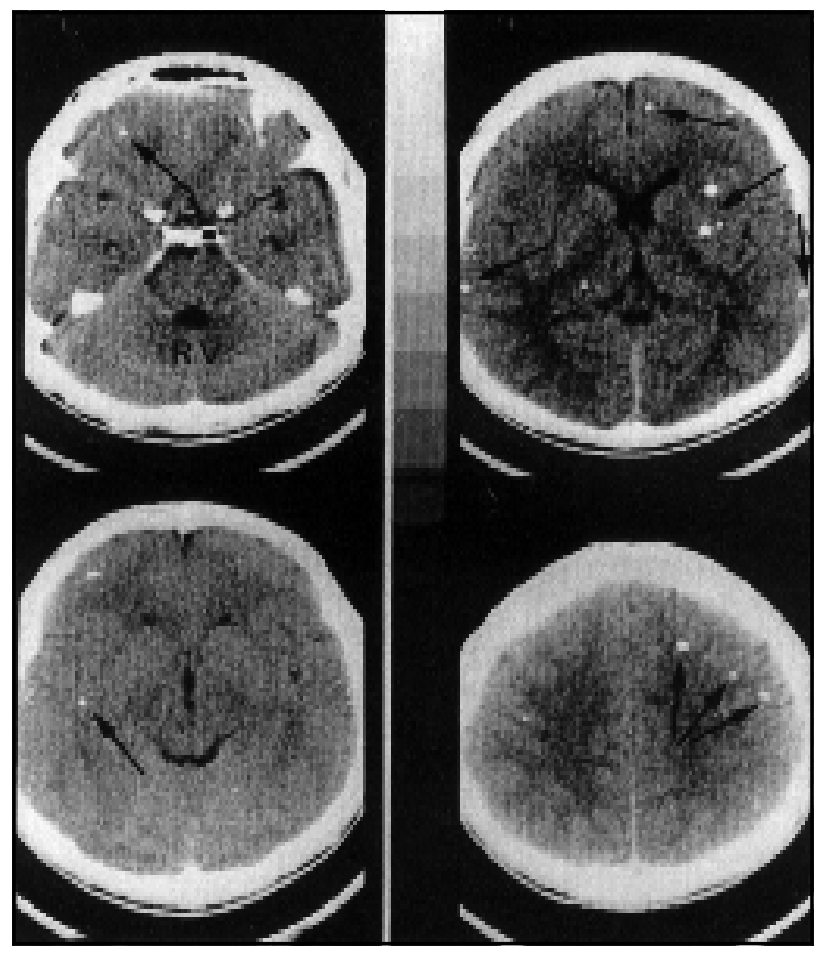

Fig 1. Características tomográficas do edema cerebral crônico pela neurocisticercose. TC de paciente masculino de 30 anos, mostrando precária diferenciação entre substancia branca e cinzenta, apagamento dos sulcos cerebrais, redução no diâmetro dos ventrículos, IVo ventrículo arredondado $(R V)$ e calcificações intracranianas (setas). 
Tabela 4. Medicações utilizadas pelos 34 pacientes com edema cerebral crônico pela neurocisticercose, antes do esquema com albendazol e no último retorno.

\begin{tabular}{lcccccc}
\hline & \multicolumn{2}{c}{ Antes do albendazol } & & \multicolumn{2}{c}{ Último retorno* } \\
\cline { 2 - 3 } \cline { 6 - 7 } Medicação & $\mathrm{n}^{\mathbf{o}}$ & $\%$ & & $\mathrm{n}^{\text {o }}$ & $\%$ \\
\hline Dextroclorofeniramina & 34 & 100,0 & & 14 & 41,2 \\
Albendazol & 32 & 94,1 & & 0 & 0 \\
Acetazolamida & 16 & 47,1 & & 5 & 14,7 \\
Anticonvulsivantes & 14 & 44,2 & & $14^{*}$ & 41,2 \\
Cetoprofeno & 12 & 35,3 & & 3 & 8,8 \\
Outras & 12 & 35,3 & & 4 & 11,8 \\
Nenhuma & 0 & 0 & & $8^{* *}$ & 23,5 \\
\hline
\end{tabular}

*doses menores que as iniciais, **incluídos 2 pacientes que não fizeram uso de ABZ.

Tabela 5. Evolução dos sintomas apresentados pelos 34 pacientes com edema cerebral crônico pela neurocisticercose.

\begin{tabular}{lcc}
\hline Evolução dos sintomas & Número de pacientes & $\%$ \\
\hline Assintomáticos & 14 & 41,2 \\
Melhora & 13 & 38,2 \\
Manutenção & 4 & 11,8 \\
Sem seguimento & 3 & 8,8 \\
\hline
\end{tabular}

Os exames bioquímicos de sangue e hemograma desses pacientes mantiveram-se dentro da normalidade, sem diferença estatisticamente significante $(\mathrm{p}>0,05)$. As reações sorológicas realizadas foram negativas.

As medicações utilizadas, antes e durante o tratamento com ABZ, bem como a manutenção delas até a última consulta, e a evolução dos sintomas apresentados pelos pacientes, antes do esquema terapêutico proposto, estão resumidas nas Tabelas 4 e 5 , respectivamente. O período de observação foi de 14 meses a 11 anos ( $\bar{x} \pm$ sd $=4,8 \pm 2,7$ anos, mediana $=3,5$ anos).

Dois pacientes apresentaram remissão dos sintomas enquanto aguardavam internação para tratamento com ABZ.

\section{COMENTÁRIOS}

O processo inflamatório imunoalérgico pode ser desencadeado tanto pela presença dos heteroantígenos como pelos autoantígenos ${ }^{8}$. Consequentemente, esse fenômeno pode ocorrer tanto próximo aos parasitas, como longe deles, ser periparasitário ou ser difuso ${ }^{9,10}$ e não se detectar a presença do cisticerco. Possivelmente fatores predisponentes e inerentes a cada indivíduo devem ser os responsáveis pela resposta, de intensidade variável, ser difusa desde o início, secundariamente difusa, como se houvesse uma cascata de reações partindo de um ou mais locais, ou ser simplesmente localizada. Nessas situações em que a resposta é difusa, além da hidrocefalia, o ECCr torna-se uma das manifestações clínicas mais representativas da NCC e merecedora de destaque.

Ressaltando a pouca ênfase que tem sido dada à manifestação de hipertensão intracraniana benigna (HICb), como uma das formas de apresentação clínica da NCC, as formas edematosas, ou 
encefalíticas $^{11-19}$, que ocorrem em $9-43 \%$ dos pacientes, têm sido relatadas como uma das apresentações da forma hipertensiva.

Na NCC, o edema pode ser a consequência não só da presença de cistos parenquimatosos, mas também constituir uma resposta anormal e global do encéfalo à pura presença de antígenos, independente da existência de formas calcificadas. O que parece é que, por algum motivo ainda não totalmente esclarecido, o sexo feminino mostra maior predisposição à manifestação de uma resposta inflamatória mais difusa que localizada ${ }^{12,16,20,21} \mathrm{ou}$, como sugere o estudo em discussão, sem a presença demonstrável de parasitas vivos no parênquima do SNC. Ou seja, a simples presença do parasita vivo não parece ser a única responsável pela resposta imunológica do hospedeiro. Parece que, grande parte das reações parasita/hospedeiro, como já foi dito, são decorrentes da imprevisível reação antígeno-anticorpo.

A síndrome de $\mathrm{HICb}^{22}$, também chamada de meningite serosa ${ }^{23}$, síndrome pseudotumoral ${ }^{24}$, hidrocefalia otítica ${ }^{25}$, hidropia meningea hipertensiva ${ }^{26}$, hidrocefalia tóxica ${ }^{27}$ ou hipertensão intracraniana benigna idiopática ${ }^{28}$, tem sido atribuída tanto ao aumento do volume sanguíneo cerebral, quanto ao edema cerebral difuso, ao aumento no volume do LCR ou à diminuição de sua absorção ${ }^{29}$. Ainda que se conheçam os vários agentes desencadeantes dessa entidade clínica ${ }^{29,30}$, continuam obscuros os mecanismos básicos de sua fisiopatogenia. Tem, como manifestações ${ }^{30-32}$ : cefaléia, alterações da visão, papiledema, ruídos intracranianos e ausência de sinais focais, sempre em pacientes alertas e orientados. O LCR geralmente mostra uma composição normal e a TC de crânio não evidencia massas intracranianas e nem aumento ventricular. Habitualmente, tem curso benigno e autolimitante ${ }^{33,34}$, predominando no sexo feminino em sua $3^{\text {a }}$ década ${ }^{31,32}$ e frequentemente está associada a obesidade ou recente ganho de peso $^{30}$. O tratamento baseia-se no uso de acetazolamida, diuréticos, punções liquóricas repetidas e derivação cérvico ou lombo-peritoneal, além de orientação para emagrecimento $^{33-36}$. O uso de corticoterapia nem sempre é indicado ${ }^{35}$, já que os corticosteróides podem ser um dos fatores desencadeantes de $\mathrm{HICb}^{36}$.

Se considerarmos a classificação de $\mathrm{HICb}^{29}$, dos 34 pacientes estudados, 20 preenchem integralmente os critérios para inclusão nesta síndrome. Sete dos outros 14 podem ser classificados como HICb atípica e, os demais 7, como HICb secundária a alterações no LCR. Ou seja, a HICb pode ocorrer sem a presença de papiledema ${ }^{29,37,38}$ ou mostrar um LCR normal ${ }^{29}$, inclusive nas medidas de pressão liquórica, como se verificou nos pacientes estudados.

$\mathrm{Na}$ casuística apresentada, pode-se observar que as manifestações do ECCr ocorrem, predominantemente, nas mulheres em sua 3르 década, como na HICb. Já, nos homens, a faixa etária mais comprometida foi a $2^{\underline{a}}$ década, proporcionalmente menor à relatada na literatura pesquisada ${ }^{31}$, ${ }^{39,40}$. Em relação à obesidade, os pacientes estudados não se comportaram conforme a literatura pesquisada ${ }^{30-32,34}$; tanto as mulheres estudadas, como os homens, mantiveram-se dentro dos limites da normalidade do peso. A explicação para esses fatos permanece tão obscura quanto a referida associação.

Assim como na HICb existem associações com determinadas condições médicas ${ }^{29}$, na ECCr, além do uso de anticoncepcionais (6 pacientes) e trauma craneoencefálico (2 pacientes), também estiveram presentes as meningites linfocitárias (4 pacientes) e as intoxicações crônicas por agrotóxicos (2 pacientes).

Como pode ser visto na Tabela 2, os sinais e sintomas na forma de ECCr correspondem, como na $\mathrm{HICb}$, a manifestações de um quadro de hipertensão intracraniana consequente, neste estudo, ao edema cerebral. Obviamente, a cefaléia foi a queixa absoluta, mas apenas em 6,2\% dos pacientes ela ocorreu isoladamente e não esteve associada a outros sintomas. Os distúrbios visuais, considerados frequentes na $\mathrm{HICb}^{12,30-32,39,41}$, ocorreram em poucos pacientes com ECCr. Assim como a síndrome epiléptica, que se manifestou como parcial, com ou sem generalização secundária, em 93,9\% dos pacientes, os distúrbios psíquicos, geralmente associados a outros sintomas ${ }^{6,12}$, constituiram uma 
das quatro queixas mais frequentes dos pacientes com ECCr. Dessa forma,talvez seja possível concluir que o ECCr, devido à NCC, possa ser considerado como mais uma entidade clínica capaz de desencadear a síndrome de $\mathrm{HICb}$.

$\mathrm{Na}$ infecção natural por cisticercos, uma boa proporção de pacientes não parece capaz de produzir anticorpos efetivos contra o parasita, sugerindo existir um quadro de imunossupressão ${ }^{3}$, uma produção de sorotipos diferentes entre os cisticercos ou, até mesmo, apresentar diferentes distribuições dos seus diversos componentes imunológicos nas variadas superfícies de localização dos parasitas ${ }^{1}$. Na prática, essa resposta imunológica humoral "não significante", pode refletir-se na negatividade das reações imunológicas que, portanto, não seriam o reflexo de uma falha na metodologia utilizada ${ }^{3}$, mas poderiam explicar as diferenças das manifestações clínicas observadas na vasta literatura sobre NCC.

O diagnóstico de NCC, não considerando as manifestações clínicas, pode ser presuntivo ou confirmativo. O primeiro é conseguido com a história clínica, epidemiologia e exames complementares como o LCR e neuroimagem. Porém, a confirmação diagnóstica só é possível, apenas, com o exame anatomopatológico de biópsias e/ou necrópsias. Frente às grandes discrepâncias, em termos de recursos laboratoriais e a necessidade em diagnosticar um quadro de NCC, o conhecimento da epidemiologia e a evolução natural da doença são, frequentemente, os únicos elementos disponíveis para a maioria dos neurologistas. Isso faz com que, muitas vezes, o diagnóstico de NCC seja definido apenas durante ou após o tratamento cisticida, pela positivação das reações imunológicas no LCR e pela melhora clínica. Esta situação ocorreu em, aproximadamente, $80 \%$ dos pacientes deste estudo, em $70 \%$ deles somente durante o esquema cisticida e, em 30\%, após o término do mesmo.

A maioria dos pacientes estudados $(91,2 \%)$ foi acompanhada ambulatorialmente por mais de 2 anos. Foi possível observar que, nessa manifestação clínica da NCC, quando a melhora não se evidencia já com o uso do anti-histamínico, ela transcorre mais lentamente e a regressão, total ou permanente, dos sinais e sintomas torna-se mais difícil. Nestes casos, a cefaléia torna-se a queixa atual menos frequente e, as manifestações epilépticas, as mais comuns.

Não se constatou qualquer evolução auto-limitante no $\mathrm{ECCr}$, como ocorre na $\mathrm{HICb}^{33,34}$, a não ser que se considere uma evolução auto-limitante, a melhora dos sintomas referidos pela metade dos pacientes com a introdução do anti-histamínico e a regressão total de seus sinais e sintomas após o uso de ABZ. Favorável a esta idéia, está a evolução satisfatória, por mais de 5 anos, de 2 pacientes que não receberam ABZ. Contrária a essa auto-limitação da evolução, estão os 6 pacientes que, embora sem queixa alguma, parecem depender da medicação sintomática utilizada,mesmo em doses sub-terapêuticas, e outros 17, que ainda apresentam sintomas em menor frequência e intensidade. Os 3 pacientes que abandonaram o seguimento, fizeram-no durante o primeiro ano pós-ABZ e estavam assintomáticos.

Ou seja, o ECCr não é manifestação rara da NCC e decorre não só da presença de múltiplos cistos parenquimatosos, mas também da possível exteriorização clínica de uma "cicatriz" imunológica de infecção pregressa, dependente da resposta do hospedeiro à presença de antígenos. Se excluirmos suas possíveis formas de evolução benigna e auto-limitante, metade dos pacientes pode permanecer com o quadro sintomatológico atenuado. O tratamento com anti-histamínico (DCF) é altamente satisfatório, embora frequentemente seja necessária sua associação com acetazolamida, algumas vezes com cetoprofeno e anticonvulsivantes ou, mais raramente, com manitol. O período de observação é prolongado, não devendo ser inferior a 4-5 anos, já que existe a possibilidade de não usar o ABZ naquelas situações em que há excelente resposta com o anti-histamínico, reforçando a idéia do ECCr, na NCC, poder constituir a manifestação clínica de uma cicatriz antigênica.

Agradecimentos - Os autores agradecem à Prof ${ }^{\mathrm{a}}$ Assistente Doutora Lídia Raquel de Carvalho, do Departamento de Bioestatística do Instituto de Biociências - Campus de Botucatu - UNESP, pela avaliação estatística dos dados apresentados. 


\section{REFERÊNCIAS}

1. Correa D, Dalma D, Espinoza B, et al. Heterogeneity of humoral immune components in human cysticercosis. J Parasitol 1985;71:535-541.

2. Flisser A, Pérez-Monfort R, Larralde C. The immunology of human and animal cysticercosis: a review. Bull WHO 1979;57:839-856.

3. Flisser A, Woodhouse E, Larralde C. Human cysticercosis: antigens, antibodies and non-responders. Clin Exp Immunol 1980;39:27-37.

4. Cruz ME, Cruz I, Preux PM, Schantz P, Dumas M. Headache and cysticercosis in Ecuador, South America. Headache 1995;35:93-97.

5. Agapejev S, Silva MD, Ueda AK. Severe forms of neurocysticercosis: treatment with albendazole. Arq Neuropsiquiatr 1996;54:82-93.

6. Agapejev S. Epidemiology of neurocysticercosis in Brazil. Rev Inst Med Trop S Paulo 1996;38:207-216.

7. Spina-França A. Síndrome liquórica da neurocisticercose. Arq Neuropsiquiatr 1961;19:307-314.

8. Livramento JA, Machado LR, Whitaker JN, Spina-França A. Proteína básica de mielina no líquido cefalorraqueano na neurocisticercose. Arq Neuropsiquiat 1985;43:160-166.

9. Pupo PP, Cardoso W, Reis JB, Silva CP. Sobre a cisticercose encefálica: estudo clínico, anátomo-patológico, radiológico e do líquido céfalo-raqueano. Arq Assist Psicop S Paulo 1945/1946;10/11:3-123.

10. Tréetiakoff C, Silva ACP. Contribuição para o estudo da cysticercose cerebral e em particular das lesões tóxicas à distância n'esta affecção. Mem Hosp Juquery 1924;1:37-66.

11. Arseni C, Samitca DC. Cysticercosis of the brain. Br Med J 1957;2:494-497.

12. Braga FM, Ferraz FAP. Forma edematosa da neurocisticercose: registro de 4 casos. Arq Neuropsiquiatr (São Paulo) 1981;39:434-443.

13. Bruck I, Antoniuk SA, Wittig E, Accorsi A. Neurocisticercose na infância: I. Diagnóstico clínico e laboratorial. Arq Neuropsiquiatr 1991;49:43-46.

14. Guerreiro MM, Facure NO, Guerreiro CAM. Aspectos da tomografia computadorizada craniana da neurocisticercose na infância. Arq Neuropsiquiatr 1992;50:343-350.

15. Minguetti G, Ferreira MCV. Computed tomography in neurocysticercosis. J Neurol Neurosurg Psychiatry 1983;46:936-942.

16. Rangel R, Torres B, Del Brutto O, Sotelo J. Cysticercotic encephalitis: a severe form in young female. Am J Trop Med Hyg 1987;36:387-392.

17. Stépien L, Choróbski J. Cysticercosis and its operative treatment. Arch Neurol Psychiat 1949;55:499-527.

18. Tolosa E. Cisticercose cerebrale: aspects cliniques et possibilités therapeutiques. Rev Neurol (Paris) 1954;90:187-208.

19. Wadia N, Desai S, Bhatt M. Disseminated cysticercosis: new observations including CT scan findings and experience with treatment by praziquantel. Brain 1988;111:597-614.

20. Del Brutto $\mathrm{OH}$, Garcia E, Talámas O, Sotelo J. Sex-related severity of inflammation in parenchymal brain cysticercosis. Arch Intern Med 1988;148:544-546.

21. Sharma K, Grupta RK. Scan-negative neurocysticercosis. Pediatr Neurosurg 1993;19:206-208.

22. Foley J. Benign forms of intracranial hypertension: "toxic" and "otitic" hydrocephalus. Brain 1955;78:1-14.

23. Quincke H. Ueber Meningitis serosa und verwandte Zustände. Dtsch Z Nervenh 1897;9:149-168.

24. Nonne M. Über Fälle von Symptomenkomplex "Tumor cerebri” mit Ausgang in Heilung (Pseudotumor cerebri). Z Nervenh 1904;27:169-216.

25. Symonds CP. Otitic hydrocephalus. Brain 1931;54:55-71

26. Davidoff LM, Dyke CG. Hypertensive meningeal hydrops:a syndrome frequently following infection in the middle ear or elsewhere in the body. Am J Ophthalmol 1937;20:908-927.

27. McAlpine D. Toxic hydrocephalus. Brain 1937;60:180-203.

28. Buchheit WA, Burion C, Haag B, Shaw D. Papilloedema and idiopathic intracranial hypertension:report of a familial occurrence. N Engl J Med 1969;280:938-942.

29. Johnston I, Hawke S, Halmagyi M, Teo C. The pseudotumor syndrome: disorders of cerebrospinal fluid circulation causing intracranial hypertension without ventriculomegaly. Arch Neurol 1991;48:740-747.

30. Giuseffi V, Wall M, Siegel PZ, Rojas PB. Symptoms and disease associations in idiopathic intracranial hypertension (pseudotumor cerebri):a case-control study. Neurology 1991;41:239-244.

31. Danzé F, Rosa A, Mizon JP.Hypertension intracrânienne bénigne (pseudo tumor cerebri): douze cas. Rev Neurol (Paris) 1984;140:37-44.

32. Wall M, George D. Idiopathic intracranial hypertension: a prospective study of 50 patients. Brain 1991;114:155-180.

33. Beatty RA. Cervical-peritoneal shunt in the treatment of pseudotumor cerebri. J Neurosurg 1982;57:853-855.

34. Sørensen PS, Krogsaa B, Gjerris F. Clinical course and prognosis of pseudotumor cerebri: a prospective study of 24 patients. Acta Neurol Scand 1988;77:164-172.

35. Johnston I, Besser M, Morgan M. Cerebrospinal fluid diversion in the treatment of benign intracranial hypertension. J Neurosurg 1988;69:195-202.

36. Walker AE, Adamkiewicz JJ. Pseudotumor cerebri associated with prolonged corticosteroid therapy. JAMA 1964;188:779-784.

37. Lipton LL, Michelson PE. Pseudotumor cerebri syndrome without papilledema. JAMA 1972;229:1591-1592.

38. Spence JD, Amacher AL, Willis NR. Benign intracranial hypertension without papilledema: role of 24-hours cerebrospinal fluid pressure monitoring in diagnosis and management. Neurosurgery 1980;7:307-336.

39. Bulens C, Meerwaldt JD, Kousdstaal PJ, van der Wildt GJ. Spatial sensitivity in benign intracranial hypertension. J Neurol Neurosurg Psychiatry 1988;51:1323-1329.

40. Seckel J, Lightman S. Cerebrospinal fluid neurohypophysial peptides in benign intracranial hypertension. J Neurol Neurosurg Psychiatry 1988;51:1538-1541.

41. Sørensen S, Trojaborg W, Gjerris F, Krogsaa B. Visual evoked potentials in pseudotumor cerebri. Arch Neurol $1985 ; 42: 150-153$. 